\title{
Novel Method for Real-Time Moiré Image Analysis Combining Two-Dimensional Entropy Theory and Quantum-Behaved Particle Swarm Optimization
}

\author{
W.J. Chen \\ Department of Industrial Education and Technology \\ National Changhua University of Education
}

\author{
Y.T. Chen \\ Department of Industrial Education and Technology \\ National Changhua University of Education
}

\begin{abstract}
This paper proposes an effective method for improving the processing quality and speed of Moiré pattern segmentation. The method involves using quantum-behaved particle swarm optimization(QPSO) based on two-dimensional (2D) entropy theory.First, the beat phenomena generated by grating interference, also called a Moiré pattern fringe, areextracted through FFT filtering. Subsequently, the fringe is segmented by two thresholds with maximized $2 \mathrm{D}$ entropy based on a QPSO algorithm. Verifying the experimental results showed that the proposed approach enabled obtaining an improved segmentation quality, fast computing performance, and favorable convergent effects.
\end{abstract}

Keywords-Moiré pattern; segmentation; binarization; twodimensional entropy; QPSO

\section{INTRODUCTION}

Shadow Moiré is a simple and powerful optical method that involves using an intuitive geometric relation of patterns to obtain information of surface points and surface profiles for objects. The method is easy to adapt and suitable for application in industry because it doesnot entail excessive costs. However, because of light source stability, space noise, and signal feedback, asshadow Moiré patterntransmitted into an optical device can result in noise light entering the detector; thus, most of the raw pattern data cannot be interpreted directly.To eliminate the noise and enhance the pattern, image processing techniques such as FFT and gradient tendency are frequently used for analyzing the data of Moiré patterns. Ping Zhong et al. [1]proposed the vary-coefficient Laplacian operation to enhance the contrast of an original Moiré fringe pattern followed by manual binarization, thinning, and repairing the enhanced image.R.C. González and R. E. Woods[2]adopted Otsu's solution by using a local intervariance estimate to determine a threshold that yielded an optimal binarization value for making binarized images clearer. Wang [3] useda particle swarm optimization(PSO) method to evaluate the threshold value of images. Thierry Pun et al.[4]presented a two-dimensional (2D)entropy method for estimating global threshold of patterns. K. Hammouche et al.[5]proposed a 2D entropy method combined with a GA optimization method for determining the threshold of maximum entropy. Hubing $\mathrm{Du}[6]$ provided an analytical method for calibrating the sensitivity of a shadow Moiré system without the need for additional calibration experiments prior to measurement. However,accurate image processing needs timeconsuming and doesnot involve estimatingthe variance at every pixel of an image. In this study, FFT and 2D maximalentropy based onquantum-behaved particle swarm optimization (QPSO)were used to extract Moiré information and rapidly analyze the stability of the segmentation method and to obtain accurate information. The process involvedcounting the pixel distribution and subsequently determining the maximal entropy at a specific pixel value for a threshold.

\section{PRINCIPLE}

Shadow Moiré is a full-field and noncontact optical measuring technique that involves using geometric interference between a reference grating and its shadow on a sample to measure relative vertical displacement at each pixel position in the resulting image.[7] As shown in Figure 1, the raysfrom the light source arrive at Point $\mathrm{A}$ and are detected by a receiver. The dashed line represents the master grid and its period is $\mathrm{g}$.

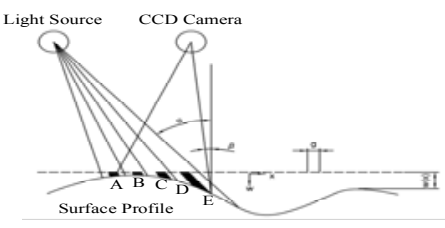

FIGURE I. SCHEMATIC OF SHADOW MOIRÉ

The shadow of the grid projected as black blocks on the object surface is the reference grid or specimen grid. Points A, $\mathrm{B}, \mathrm{C}$, and $\mathrm{D}$ represent the position of the light arriving at the object. The variable $\alpha$ is the angle of the incident, and $\beta$ is the angle of the reflection with respect to the normal direction of the reference grating. The variable $\mathrm{W}$ is the depth between the object and the grating that is measured by the number of Moiré fringes $\mathrm{N}$ between two particular points on the object. Through geometric analyzing, the mathematical formulationcan be expressed as follows.

$$
\mathrm{W}=\frac{\mathrm{N}}{\tan \alpha+\tan \beta} \mathrm{g}
$$




\section{PATTERN PRE-PROCESSING}

The pre-processing procedure includes a Laplacian operator and low-pass filterfrom FFT that are used to extract the Moiré fringe. Equation (2) is the Laplacian operator used for edge extraction of an image. The imagefeatures2-D information; consequently, the Laplacian equation also features two dimensions.

$$
\begin{gathered}
\nabla^{2} \mathrm{I}=\frac{\partial \mathrm{I}}{\partial \mathrm{x}}+\frac{\partial \mathrm{I}}{\partial \mathrm{y}} \\
\mathrm{F}(\mathrm{u}, \mathrm{v})=\sum_{\mathrm{x}=0}^{\mathrm{M}-1} \sum_{\mathrm{y}=0}^{\mathrm{N}-1} \mathrm{I}(\mathrm{x}, \mathrm{y}) \mathrm{e}^{-\mathrm{j} 2 \pi\left(\frac{\mathrm{ux}}{\mathrm{M}}+\frac{\mathrm{vy}}{\mathrm{N}}\right)} \\
\mathrm{I}(\mathrm{x}, \mathrm{y})=\sum_{\mathrm{u}=0}^{\mathrm{M}-1} \sum_{\mathrm{v}=0}^{\mathrm{N}-1} \frac{1}{\mathrm{MN}} \mathrm{F}(\mathrm{u}, \mathrm{v}) \mathrm{e}^{\mathrm{j} 2 \pi\left(\frac{\mathrm{ux}}{\mathrm{M}}+\frac{\mathrm{vy}}{\mathrm{N}}\right)}
\end{gathered}
$$

According to(2), the FFT operation is used for a lowfrequency tendency of these extracted edges of an imageand removing the noise from the image. Let $\mathrm{I}(\mathrm{x}, \mathrm{y})$ in Eq. (3) be animage before transformation and $\mathrm{x}$ and $\mathrm{y}$ be the spatial position. $\mathrm{M}$ and $\mathrm{N}$ are the size of an image, $\mathrm{u}$ and $\mathrm{v}$ are the position of thefrequency domain. The variable $\mathrm{F}(\mathrm{u}, \mathrm{v})$ represents the spectrum in the frequency domain. The variable I' in Eq. (4) is a low-pass image of the frequency domain that is a pure low-frequency image andsuperior tothat of the spatial domain.

\section{TWO-DIMENSIONAL ENTROPY SEGMENTATION}

Two-dimensionalentropy [8] represents a particular entropy theory used in image processing. One of the dimensions is related to every pixel;the second dimension is related to its eight neighboring pixels, and the third dimension is count-related to the other two dimensions; they thus form a 3-D histogram of pixels, which is used for calculating 2-D entropy. A top view of a3D histogram is shown in Figure 2,

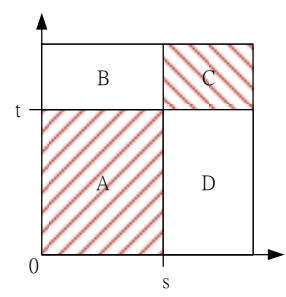

FIGURE II. TOP VIEW OF A 3D HISTOGRAM

where $\mathrm{s}$ is a threshold for the center pixel in a local ninesquare region, and $\mathrm{t}$ is another threshold for its eight neighboring pixelsaverage and its range in $[0,255]$ when using an 8-bit image. In a typical image, the tendency of pixel distribution is concentrated at the diagonal, involved in A and C.The $\mathrm{B}$ and $\mathrm{D}$ regionsfeature an extreme contrast between the center pixel and its eight neighboring pixels,indicating an unnatural region. Entropy theory can be appliedto determine the threshold and the maximal amount of valuable information in an image. The 2-D entropy is defined as follows.

$$
\mathrm{H}_{2 \mathrm{D}}=\log (\mathrm{P}(\mathrm{A}))(1-\mathrm{P}(\mathrm{A}))+\frac{\mathrm{H}(\mathrm{A})}{\mathrm{P}(\mathrm{A})}+(\mathrm{H}(\mathrm{L})-\mathrm{H}(\mathrm{A}))(1-
$$

where $P(A)=\sum_{i=0}^{s} \sum_{j=0}^{t} p_{i j}$ is the summation of probabilityfor event Aand $\mathrm{s} H(\mathrm{~A})=\sum_{\mathrm{i}=0}^{\mathrm{s}} \sum_{\mathrm{j}=0}^{\mathrm{t}} \mathrm{p}_{\mathrm{ij}} \log _{\mathrm{b}} \mathrm{p}_{\mathrm{ij}}$ is 2-D Entropy in region $A$. $H(C)=\sum_{i=s+1}^{L-1} \sum_{j=t+1}^{L-1} p_{i j} \log _{b} p_{i j}$ is 2-D Entropy of $\mathrm{C}$ region and $\mathrm{H}(\mathrm{L})=\sum_{\mathrm{i}=0}^{\mathrm{L}-1} \sum_{\mathrm{j}=0}^{\mathrm{L}-1} \mathrm{p}_{\mathrm{ij}} \log _{\mathrm{b}} \mathrm{p}_{\mathrm{ij}}$ is 2-D Entropy includes the information of $\mathrm{A}, \mathrm{B}, \mathrm{C}$, and D. In addition, $\mathrm{i}, \mathrm{j}$ is the index variable and pij is the probability of occurrence of the pixel pair, center pixel and the average grey value of its 8neighborhood points.

\section{OVERVIEW OF PARTICLE SWARM \\ OPTIMIZATIONANDQUANTUM-BEHAVED PARTICLE SWARM OPTIMIZATION}

The PSO algorithm is a fast optimization method based on bio-inspired dynamic behavior.[9][10] It imitates biological clustering, flying, and search processes. However, a classical PSO algorithm cannot converge to a global solution in a search space. S. Jun[11]used the QPSOalgorithmto addressthis disadvantage. Updating the position inQPSO algorithminvolves considering only the position of potential adequately described according to a probability density function. Therefore, QPSO algorithms exhibit a wide search capacity and have fewer loopsin updatinga particle's position.The QPSO algorithm is proposed as (6)-(8)

$$
\begin{aligned}
& \mathrm{p}^{\mathrm{i}}=\frac{\phi_{1} * \mathrm{p}_{\text {local }}^{\mathrm{i}}+\phi_{2} * \mathrm{p}_{\text {global }}}{\phi_{1}+\phi_{2}} \\
& \mathrm{~L}=\left(\frac{1}{\mathrm{c}}\right) *\left|\frac{\sum \mathrm{p}_{\text {local }}^{\mathrm{i}}}{\mathrm{m}}-\mathrm{x}^{\mathrm{i}}(\mathrm{t})\right| \\
& \mathrm{x}^{\mathrm{i}}(\mathrm{t}+1) \leftarrow \mathrm{p}^{\mathrm{i} \pm \mathrm{L} * \ln \left(\frac{1}{\mathrm{u}}\right)}
\end{aligned}
$$

where $\phi_{1}, \phi_{2}$ and $\mathrm{u}$ are uniformly distributed random number. $\mathrm{m}$ is the total number of particles. $\mathrm{p}$ represents the attraction center of potential well, $\mathrm{p}_{\text {local }}^{\mathrm{i}}$ is the best position of ith particle experienced, $p_{\text {global }}$ is the best position of all particles experienced and weighted by $\phi_{1}$ and $\phi_{2}$, respectively. $\mathrm{L}$ is the characteristic length of potential well and $\mathrm{c}$ is a constant which always specified large than 1 to guarantee the convergence of QPSO. When the L is determined, the position of each particle $\mathrm{x}^{\mathrm{i}}$ is updated through (8) and the symbol " $\leftarrow$ " is used to show next iteration.

\section{RESEARCH PROCEDURE}

In this study, the pre-processing steps of the original Moiré fringe include a Laplacian operation and alow-pass operation of FFT. The subsequent steps involve segmentation based on 2D entropy criteria with QPSO fordeterminingthe maximalentropy to segment a low-pass image. The total processing procedure is shown in Figure 3. 


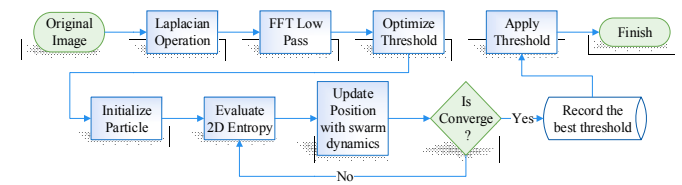

FIGURE III. FLOW CHART OF RESEARCH PROCEDURE

\section{RESUlTS AND ANALYSIS}

According to(2) and (3), Figure 4(a) and (b) display the original Moiré fringes and the results of the Laplacian operation.Figure 4 (c) and (d) represent the results of FFT before and after the low-pass operation in the frequency domain. The truncated image was used to perform the reverse transform of FFT and obtainthe image I' in the spatial domain, as shown in Figure 4 (e). Finally, the segmentation result is illustrated in Figure 4 (f).

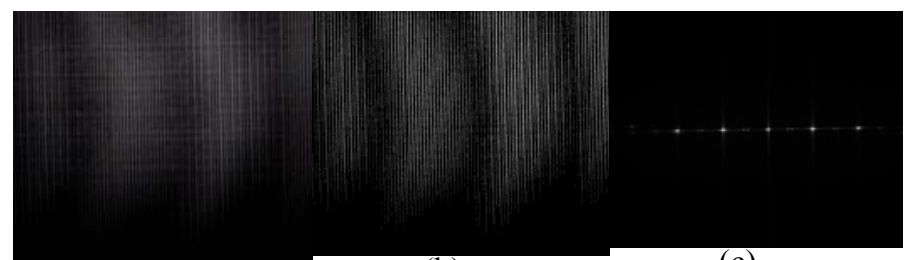

(a)

(b)

(c)

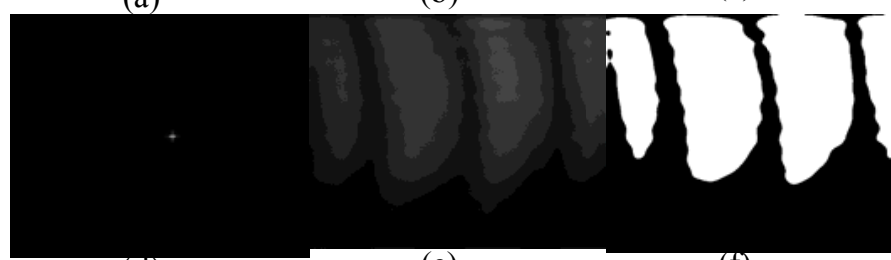

(d)

(e)

(f)

FIGURE IV. (A) ORIGINAL IMAGE. (B) RESULT OF LAPLACIAN OPERATION. (C) FOURIER SPECTRUM OF (B). (D) FOURIER FILTERING. (E) IMAGE AFTER FILTERING. (F) SEGMENTATION RESULT

Figures 5 (a)-(c)and 5 (d)-(f)represent the results of the PSO and QPSO algorithms that exhibited unstable and stable 2-Dentropy, respectively. They show the solving performance of maximizing 2-D entropy forMoiré imageswith 20 iterations and conducting 50 experiments. The curvesof differentcoloursmean that the results were produced by different experiments under the same conditions. (a)

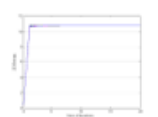

(d)

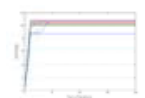

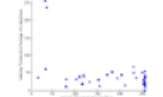

(b)

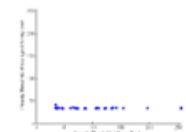

(e)

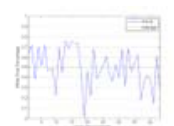

(c)

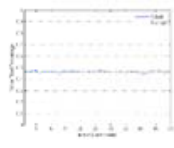

(f)

FIGURE V. COMPARISON BETWEEN PSO IN (A)-(C) AND QPSO IN (D)-(F)

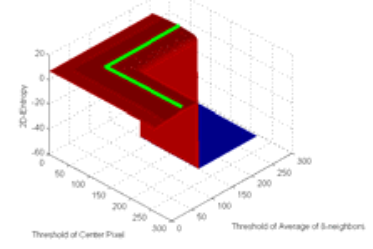

FIGURE VI. FORCED 2D ENTROPY SOLUTIONS IN ALL PARAMETER PAIRS

In addition, whenthe iteration numberwas adjustedtomaintain stable resultsforthe PSO and QPSO, the PSO expended more time than did the QPSO. Therefore, the convergence advantage of the QPSO can ensure that the proposed approach is used in a real-time scenario for extracting the Moiré fringe pattern.Finally, the validity of the parameters was confirmedthrougha forced solution. The parameter pair that was used for segmentation is discussed in the 2-D entropy segmentation section. The parameter pairs were calculated by theQPSO algorithm, which is presented inFigure 5 (e); every point was a result produced by a full-field calculation, and the forced 2-D entropy solutions in all parameter pairs (approximately $256 \times 256$ ) are shown in Figure (6). An axis of Figure (6) corresponds to the abscissa of Figure 5 (b) and (e); the other one corresponds to the ordinate, and the final one corresponds to the 2-D entropy result calculated by the parameter pair.Figure 5 (c) shows that the PSO caused an unstable and poor segmentation result.

The white-region percentage after segmentation exhibited substantial fluctuation in various iterations. The distribution of the parameter pair calculated by the QPSO, as shown inFigure 5 (f), exhibited the same tendency as the forced 2D entropy solution. In variousiterations, the QPSO exhibiteda global search capability to obtain maximal 2-D entropy in the segmentation case and ensured obtaining stable segmentation results.

\section{CONCLUSION}

The Moiré method is a powerful measurement tool, especially regarding surface properties of material depending on strain and warpage. This technique has been widely used in numerousindirect measuring scenariosto extract valid information of surface for object. Therefore, the proposed approach in this study can be used to perform a segmentation process stably and fast to extract the Moiré pattern clearly and to facilitate real-time analysis.

\section{REFERENCE}

[1] P. Zhong, S. Wang, Y. Jin, X. Tu, and N. Luo, "A method of image preprocessing based on nonlinear diffusion and information extraction," Comput. Math. Appl., vol. 61, pp. 2132-2137, Apr. 2011.

[2] R. C. González and R. E. Woods, Digital image processing. Prentice Hall, 2008.

[3] Wang Feng-chao, "An Improved 2-D Maximum Entropy Threshold Segmentation Method Based on PSO," in 2nd International Congress on Image and Signal Processing, 2009. CISP '09, 2009, pp. 1-5.

[4] T. Pun, "A new method for grey-level picture thresholding using the entropy of the histogram," Signal Process., vol. 2, no. 3, pp. 223-237, Jul. 1980 .

[5] K. Hammouche, M. Diaf, and P. Siarry, "A multilevel automatic 
thresholding method based on a genetic algorithm for a fast image segmentation," Comput. Vis. Image Underst., vol. 109, no. 2, pp. 163 175, Feb. 2008.

[6] [6] H. Du, J. Wang, H. Zhao, and P. Jia, "Calibration of the high sensitivity shadow moiré system using random phase-shifting technique," Opt. Lasers Eng., vol. 63, pp. 70-75, 2014.

[7] D. Post, B. Han, and P. Ifju, High Sensitivity Moire: Experimental Analysis for Mechanics and Materials. Springer, 1994.

[8] A. S. Abutaleb, "Automatic thresholding of gray-level pictures using two-dimensional entropy," Comput. Vis. Graph. Image Process., vol. 47, no. 1, pp. 22-32, 1989.

[9] J. Kennedy and R. Eberhart, "Particle swarm optimization," in , IEEE International Conference on Neural Networks, 1995. Proceedings, 1995, vol. 4, pp. 1942-1948.

[10] S. M. Mikki and A. A. Kishk, "Quantum Particle Swarm Optimization for Electromagnetics," IEEE Trans. Antennas Propag., vol. 54, no. 10, pp. $2764-2775$, Oct. 2006.

[11] Jun Sun, Bin Feng, and Wenbo Xu, "Particle swarm optimization with particles having quantum behavior," in Congress on Evolutionary Computation, 2004. CEC2004, 2004, vol. 1, pp. 325-331. 